\title{
Tipo de fibra muscular y su relación con el abordaje fonoaudiológico en los trastornos de la deglución
}

\author{
Gabriel González a,* \\ ${ }^{a}$ Carrera de Fonoaudiología, Universidad Pedro de Valdivia, Chile
}

\begin{abstract}
RESUMEN
Los actuales protocolos de intervención en deglución no especifican parámetros de los ejercicios tales como la cantidad de series, duración, tiempos de repetición, entre otros. Lo anterior, es un desafío para la intervención fonoaudiológica en los trastornos de la deglución. El objetivo de este trabajo es acercar al profesional fonoaudiólogo al conocimiento de las fibras musculares, ya que es un conocimiento que se debe considerar antes de indicar ejercicios musculares cráneo-cérvico-orales. Por ello, se describen las principales fibras musculares con sus respectivas características fundamentales, como lo son: la resistencia a la fatiga y la velocidad de contracción. Se revisa la literatura sobre la dispersión de las fibras musculares, de algunos de los principales músculos que participan en el proceso deglutorio. También, se analizan diversas particularidades de los músculos de la zona cráneo-cérvico-oral. Se describen además las diferentes difícultades para evaluar esta musculatura. Finalmente, se expone la relevancia práctica de conocer estos tipos de fibras musculares y las perspectivas futuras de este enfoque basado en parámetros del ejercicio y la medición de variables objetivas.
\end{abstract}

\section{Type of muscle fibre and its relationship to the Speech-Language Pathology approach in swallowing disorders}

\begin{abstract}
Swallowing intervention protocols in dysphagia do not specify the parameters of the exercises, namely the duration, frequency and number of series, all of which present a challenge in speech therapy intervention in swallowing disorders. The purpose of this review is to provide Speech and Language Pathologists with background information concerning crucial muscle fibres used in swallowing therapy and to subsequently indicate appropriate skull-cervical-oral muscle exercises. We describe the primary muscle and its characteristics such as fatigue resistance and contraction speed, review the types of fibres of some of the main muscles that participate in the swallowing process, and present the particularities of the muscles of the craniocervical-oral area in comparison with the rest of the skeletal muscles. We also provide information regarding the difficulties in evaluating the musculature involved in the swallowing process before finally highlighting the relevance of understanding the roles and characteristics of these muscles for clinical practice.
\end{abstract}

\section{Palabras clave:}

Fibra muscular; Disfagia;

Ejercicio muscular

\section{Keywords:}

Muscle fibre; dysphagia; Muscle exercise

\footnotetext{
*Autor/a correspondiente: Gabriel González

Email:fonogabriel@gmail.com
} 
La literatura científica expone diversos procedimientos terapéuticos para enfrentar los trastornos de la deglución. En los últimos 10 años, cada vez es más común encontrar programas terapéuticos para tratar la disfagia orofaríngea centrados en el ejercicio. Un ejemplo de este tipo de intervenciones, es el programa "McNeill Dysphagia Therapy Program MDTP" (Crary et al., 2012), que está basado en su totalidad en la comprensión de la fisiología del ejercicio de la musculatura orofacial. Este programa, utilizado por el profesional fonoaudiólogo, no considera una sola técnica que se repite en el tiempo, si no que propone una progresión de tareas centradas en el rendimiento individual de cada persona, para mejorar la fuerza, velocidad y coordinación del mecanismo deglutorio alterado (Carnaby et al., 2019). Sin embargo, el profesional fonoaudiólogo tiene múltiples dificultades para medir los resultados en el tono y/o en la fuerza muscular en los pacientes con dificultades de la deglución. Ello, debido a las limitaciones para objetivar las medidas que permitan evaluar tanto el nivel inicial como los avances a nivel de tono y fuerza muscular de las personas que están siendo tratadas. Adams et al. (2013) a partir de un metanalisis sobre el tema destaca la necesidad de cuantificar el rendimiento muscular de los pacientes a intervenir.

En relación con las estrategias clásicamente utilizadas para la evaluación de la contracción muscular, las más utilizadas son las aplicadas a nivel de las extremidades. Ellas corresponden a la palpación y/o a la percepción de la resistencia muscular a la movilización pasiva (Chu \& Barlow, 2016). Es plausible proponer utilizar estas mismas estrategias en la evaluación de la musculatura cráneo cervical. Sin embargo, ello no es aconsejado por varias razones. En primer lugar, las estrategias de evaluación de las extremidades presentan limitaciones importantes dado que su metodología de valoración es en su mayoría perceptual, ya sea utilizando criterio clínico o escalas de valoración perceptual. Este tipo de evaluación ha sido criticada por la ausencia de criterios de rigor científico y la insuficiente evidencia científica que las sustenta (Susanibar et al., 2016). Además, son dependientes de la experiencia del terapeuta que evalúa (Sciote et al., 2003). En segundo lugar, la valoración de la musculatura realizada en las extremidades no puede ser transferida a la zona cráneo cérvico oral (CCO) (Chu \& Barlow, 2016), debido a que esta zona presenta características distintas a las de la musculatura de las extremidades.

Dado lo anteriormente expuesto, se propone que un adecuado conocimiento de los diferentes comportamientos fisiológicos de la musculatura, considerados en los tratamientos de los trastornos de la deglución, permitiría al profesional fonoaudiólogo desarrollar estrategias más objetivas de evaluación e intervención de la musculatura deglutoria. El propósito de esta revisión es proporcionar información relevante sobre los tipos de fibras musculares, con el fin de destacar los aspectos que se deben considerar antes de indicar ejercicios musculares de la zona CCO.

En primer lugar, se revisarán los diferentes tipos de fibras musculares que se pueden encontrar en el cuerpo humano, con el objetivo de entender las diferencias en el funcionamiento muscular, dependiendo de la fibra que compone la musculatura. Luego, se describirán los tipos de fibras presentes en la musculatura asociada al proceso deglutorio. Posteriormente, se expondrán las particularidades presentes en la musculatura $\mathrm{CCO}$ en comparación con el resto de la musculatura esquelética. Esto permitirá entender por qué no se pueden extrapolar las estrategias utilizadas para evaluar la musculatura de las extremidades en el abordaje de la musculatura de la zona CCO. En el punto siguiente se abordará la relación entre el tipo de fibra muscular y el funcionamiento de la musculatura relacionada con el proceso deglutorio. Ello, con el objetivo de entregar luces sobre que parámetros esperar en cada grupo muscular, lo que debe ser tomado en cuenta en la evaluación e intervención de dicha musculatura. Finalmente, se discutirá la implicancia que este conocimiento tiene a nivel clínico para el quehacer fonoaudiológico.

\section{TIPO DE FIBRAS MUSCULARES}

La musculatura esquelética es versátil y se somete a un amplio rango de demandas funcionales. A nivel macroscópico el músculo esquelético está conformado por fibras musculares. A su vez, estas fibras están conformadas por unidades más pequeñas. A nivel microscópico las fibras musculares se componen de células con distintas propiedades tanto a nivel metabólico como molecular (López \& Fernández, 2006). Las fibras musculares, se diferencian en diversos aspectos como son: diámetro, tolerancia a la fatiga, sistema energético predominante, entre otros. A modo de resumen, en la Tabla 1, se describen las principales características diferenciadoras.

En los mamíferos se reconocen cuatro tipos de fibras musculares: fibras tipo I (de contracción lenta), y fibras tipo II (de contracción rápida). A su vez, las fibras tipo II se dividen en 3 subtipos: IIA, IIX y IIB (López \& Fernández, 2006). En el ser humano únicamente encontramos fibras musculares tipo: I, IIA y IIX. Se ha comprobado que las fibras musculares que antes se describían como IIB, son en realidad fibras IIX (Bottinelli \& Reggiani, 2000; Canepari et al., 2010). Esta información es relevante, ya que en la literatura previa al año 2000, cuando se describen las fibras tipo 
IIB en humanos, en estricto rigor, se está dando cuenta de fibras tipo IIX. La característica de cada fibra muscular es diferenciada entre su velocidad de contracción, así como su resistencia (McArdle et al., 2010). A continuación, se detallan las principales características de las fibras musculares:

Fibras Tipo I: son fibras lentas, porque son las que más pausadamente hidrolizan el ATP para contraerse. Así mismo estas fibras poseen una gran capacidad oxidativa, debido a su baja absorción de calcio y su lenta propagación de velocidad del impulso nervioso. Todas estas características las convierten en fibras bien adaptadas para la realización de ejercicios aeróbicos y prolongados (por ejemplo: Maratón). En resumen, se describen como de contracción lenta y de alta resistencia a la fatiga muscular (López \& Fernández, 2006).

Fibras Tipo II: presentan una velocidad de contracción mucho mayor que las fibras tipo I. Incluso se describe que las tipo II se contraen entre 3 y 5 veces más rápido que las tipo I (Bottinelli \& Reggiani, 2000). Las fibras tipo IIX son las que presentan mayor velocidad de contracción, mientras que las IIA manifiestan una velocidad intermedia (López \& Fernández, 2006). Las características fisiológicas del tipo II permiten almacenar más cantidad de calcio, lo que facilita la conducción nerviosa de las miofibrillas, con el fin que sean cada vez más veloces. Los autores López \& Fernández (2006) concluyen que las fibras tipo II son las que obtienen una respuesta más veloz, con mayor tensión y que su metabolismo las hace rápidamente fatigables. Así, parecen particularmente adaptadas a participar en actividades físicas breves e intensas. Por ejemplo, el levantamiento de pesas.

Tabla 1. Características diferenciadoras de los distintos tipos de fibras musculares.

\begin{tabular}{lccc}
\hline & $\begin{array}{c}\text { Lentas } \\
\text { (Tipo I) }\end{array}$ & $\begin{array}{c}\text { Intermedias } \\
\text { (Tipo IIA) }\end{array}$ & $\begin{array}{c}\text { Rápidas } \\
\text { (Tipo IIX) }\end{array}$ \\
\hline Diámetro & Intermedio & Grande & Pequeño \\
Resistencia a la Fatiga & Alta & Intermedio & Bajo \\
Velocidad de Contracción & Lenta & Rápida & Rápida \\
$\begin{array}{l}\text { Actividad ATP } \\
\begin{array}{l}\text { Sistema Energético } \\
\text { predominante } \\
\text { Motoneurona }\end{array}\end{array}$ Aeróbico & Combinado & Anaeróbico \\
Descarga & Pequeña & Grande & Grande \\
\hline
\end{tabular}

Fuente: López \& Fernández (2006).

\section{MÚSCULOS QUE PARTICIPAN EN LA DEGLUCIÓN Y SU RESPECTIVA DISPERSIÓN DE FIBRAS MUSCULARES}

Existe una gran cantidad de grupos musculares que el Fonoaudiólogo debe conocer para llevar a cabo una correcta práctica clínica en el área de la deglución. Particularmente, es necesario conocer las principales características histoquímicas según fibra muscular, de los músculos que participan en la deglución. A continuación, se describen las características histoquímicas de los músculos más importantes para el proceso deglutorio (Cichero \& Murdoch, 2006), en relación con las etapas deglutorias clásicas. Estas son: (1) etapa preoral/oral: orbicular de los labios, geniogloso, temporal. (2): faríngea: digástrico, milohioideo, constrictor faríngeo. (3) Esofágico: cricofaríngeo.

\section{Etapa preoral/oral}

Orbicular de los labios: Evidencia actual muestra que el músculo del orbicular de los labios tiene una distribución en su parte labial de un $22 \%$ de fibras tipo I y un $73 \%$ de fibras tipo II. En la parte marginal la distribución consiste en un $30 \%$ de fibras tipo I y un $66 \%$ de fibras tipo II (Hwang et al., 2011).

Geniogloso: En la porción anterior, se observa una dispersión de $31 \%$ de fibras tipo I y un $69 \%$ de fibras tipo II (Saigusa et al., 2001). En la porción posterior estas diferencias se equiparán, obteniendo un $51 \%$ de fibras tipo I y un $49 \%$ de fibras tipo II.

Temporal: El músculo temporal posee principalmente fibras tipo I. Son muy abundantes en la zona profunda, donde representan casi el $80 \%$ del total. En la zona superficial las fibras tipo I y las tipo IIX, están aproximadamente en la misma proporción (Martín, 1999). Sin embargo, otros autores describen que en la zona superficial existe más tipo IIX (57\%) que tipo I (40\%) (Eriksson \& Thornell, 1983).

\section{Etapa faríngea}

Digástrico: Existe una proporción de fibras de un 29,7\% de fibras tipo I y un $61 \%$ de fibras tipo II (44,2 IIA y 17,8 IIX). El porcentaje restante se redistribuye en fibras hibridas (Korfage et al., 2000).

Milohioideo: La dispersión de las fibras musculares de esta zona, varía entre un $42 \%$ fibras tipo I, y un 55\% fibras tipo II, las demás fibras (hibridas y fetales) toman el porcentaje restante (Korfage et al., 2000).

Constrictor de la Faringe: En un principio (Leese \& Hopwood, 1986) se describió que solo el $32 \%$ de las fibras son tipo I. 
Posteriormente (Sundman et al., 2004) se describió una dispersión de $36 \%$ fibras tipo I y $43 \%$ fibras tipo II. La proporción restante se subdivide en fibras hibridas.

\section{Etapa esofágica}

Cricofaringeo: Las primeras evidencias sobre este músculo describen que posee un $82 \%$ de fibras tipo I (Kristmundsdottir et al., 1990). Datos más actuales describen que este músculo presenta una distribución más equitativa entre cada tipo de fibra, aunque con mayor frecuencia de fibras tipo I (Sundman et al., 2004).

En relación al análisis de la evidencia en la literatura, se resumen en la Tabla 2 todos los grupos musculares presentados con su respectiva velocidad de contracción y nivel de fatigabilidad.

Tabla 2. Fibras musculares, según su velocidad de contracción y nivel de fatigabilidad.

\begin{tabular}{|c|c|c|c|}
\hline & $\begin{array}{l}\text { Lentas } \\
\text { (Tipo I) }\end{array}$ & $\begin{array}{c}\text { Intermedias } \\
\text { (Tipo IIA) }\end{array}$ & $\begin{array}{l}\text { Rápidas } \\
\text { (Tipo IIX) }\end{array}$ \\
\hline Diámetro & Intermedio & Grande & Pequeño \\
\hline Resistencia a la Fatiga & Alta & Intermedio & Bajo \\
\hline $\begin{array}{l}\text { Velocidad de } \\
\text { Contracción }\end{array}$ & Lenta & Rápida & Rápida \\
\hline Actividad ATP & Baja & Alta & Alta \\
\hline $\begin{array}{l}\text { Sistema Energético } \\
\text { predominante }\end{array}$ & Aeróbico & Combinado & Anaeróbico \\
\hline Motoneurona & Pequeña & Grande & Grande \\
\hline Descarga & Baja & Alta & Alta \\
\hline
\end{tabular}

Fuente: Elaboración propia.

\section{PECULIARIDADES DE LA MUSCULATURA DE LA ZONA CRÁNEO-CÉRVICO-ORAL (CCO)}

Como ya se mencionó, a diferencia de la musculatura de las extremidades, los músculos de la zona CCO tiene sus propias características peculiares (Osterlund et al., 2011). Debido a lo anterior, resulta dificultoso y, en ocasiones imposible, evaluar e intervenir un músculo de manera aislada, sin el movimiento accesorio o antagonista de otros músculos aledaños.

A continuación, se describen algunas de estas características musculares de la zona CCO:
- Son escasas las estructuras musculares orales que pueden ser palpadas o movilizadas pasivamente, dado su tamaño y orientación.

- La relación entre los músculos antagonistas y agonistas de la región orofacial es escasa, a diferencia de las extremidades.

- La gran mayoría de los músculos orofaciales no están orientados a lo largo de los ejes del esqueleto, lo que se da en las extremidades. En algunos de los músculos donde se observa lo anterior son los de la mímica y los velofaríngeos

- La distribución de los tipos de fibra muscular es variable, incluso en el mismo proceso muscular. Por ejemplo, la lengua tiene más fibras tipo I en la raíz y fibras tipo II en su ápice (Susanibar et al., 2016). Otros músculos como el masetero presentan fibras denominadas híbridas, ya que expresan dos o más isoformas (Sciote et al., 2003).

- Existen músculos orofaciales que no tienen inserción ósea, a diferencia de los músculos de las extremidades. Por ejemplo, Orbicular de los Labios (Susanibar et al., 2016).

- La contracción muscular de la zona CCO varía. Por ejemplo, las fibras tipo I del masetero son lentas. Mientras que las fibras de los músculos laríngeos (de tipo II) se contraen con mayor rapidez (Sciote et al., 2003).

- Algunos músculos, como por ejemplo los transversos y longitudinales linguales son hidrostatos musculares (Stone \& Murano, 2007). Por ello, generalmente mantienen su mismo volumen a pesar de estar en fase de contracción. Este fenómeno es distinto en los músculos de las extremidades, en donde al contraerse disminuyen su diámetro.

- Existe un orden distinto de la proyección de fibras musculares en la zona $\mathrm{CCO}$, muestra de esto puede ser el músculo temporal. Dicho músculo posee una proyección céfalo-caudal de sus fibras en su parte anterior. Sin embargo, a medida que se avanza hacia la parte posterior, la proyección de sus fibras se horizontaliza, describiendo una proyección antero-posterior (Fuller et al., 2012).

- El proceso propio del envejecimiento de la musculatura de la zona CCO, también tiene sus propias singularidades (Susanibar et al., 2016). Esto podría explicar el motivo de que la lengua de las personas mayores, no se comporta de la manera esperada a las características en el proceso del envejecimiento. Ello debido a que sus diferencias de tono muscular son muy similares en adultos jóvenes, fenómeno que es distinto en los músculos de las extremidades (Dietsch et al., 2015). 


\section{RELACIÓN ENTRE TIPO DE FIBRA Y FUNCIONAMIENTO MUSCULAR EN LA ZONA CCO Y SU IMPLICANCIA PARA EL PROCESO DEGLUTORIO}

La información anteriormente expuesta muestra la fisiología muscular con el fin de comprender la dispersión de las fibras musculares de los músculos de la zona CCO.

A continuación, se intentará explicar el comportamiento del músculo o grupo muscular dependiendo del tipo de fibra muscular que lo componga y su implicancia para el proceso deglutorio:

- Musculatura orofacial de fibras rápidas (principalmente IIX y IIA): Dentro de este grupo se encuentran músculos con funciones orales, tales como: orbicular de los labios y digástrico, dentro del músculo orbicular de los labios. Se describe que casi el $70 \%$ de este músculo está compuesto por fibras tipo II, por lo que, cumple una función fásica en su mayoría. Esto determina que es altamente fatigable, pero su velocidad de contracción lo convierte en uno de los músculos más rápidos de la zona $\mathrm{CCO}$. Es útil en el proceso de deglución/habla, ya que en este proceso se requiere movimientos orales rápidos y precisos (Hwang et al., 2011). Dentro del músculo digástrico, se encuentra una alta proyección de fibras tipo II (61\%). Dicho músculo también estaría cumpliendo funciones fásicas, lo que requiere pocos tiempos de estimulación, con mayores cargas. Esta dispersión de fibras en ambos músculos determina que requieren una rápida aceleración, debido a las necesidades de los movimientos orofaciales que realizan (Kent, 2010).

- Musculatura orofacial de fibras hibridas rápidas (proporción más equitativa entre IIX, IIA y I): Dentro de este grupo, debido a su composición de fibras, se encuentran músculos faríngeos, tales como: Constrictor de la faringe, Milohioideo y Geniogloso. Realizan movimientos principalmente rápidos, pero al tener algunas porciones de fibras tipo I, tendrán cierta resistencia a la fatiga. Se ha descrito que la zona faríngea es uno de los lugares con mayores variabilidades individuales, encontrando algunos movimientos y resultados inusuales (Kent, 2010). Estos grupos musculares también necesitan altas cargas de trabajo con tiempos un poco más extensos, en comparación con los músculos denominados rápidos.

- Musculatura orofacial de fibras hibridas intermedias (principalmente IIA y I): En este grupo muscular se encuentra el músculo temporal, que es uno de los principales músculos masticatorios (Fuller et al., 2012). Su dispersión de fibras se describe como un hibrido, debido a que su repartición de fibra muscular es diferente en cada parte del músculo (Kent, 2010). Estas características histoquímicas, de tipo relativamente lentas, facilitan el apoyo postural y el habla, favoreciendo el cierre bucal y retrasando la fatiga en el acto de deglución o del habla.

- Musculatura de fibras Lentas (principalmente I y IIA): Dentro de este grupo está el músculo cricofaríngeo, el cual participa en la fase esofágica de la deglución, favoreciendo la dilatación del esfínter esofágico superior. Este músculo cumple funciones más tónicas, ya que posee una alta resistencia a la fatiga (Kristmundsdottir et al., 1990). Debido a la función fisiológica del cricofaríngeo, generalmente se encuentra en contracción, porque solo se dilata en el acto de la deglución. Por lo tanto, este estado de constante de contracción requiere de una alta dispersión de fibras tipo I.

A continuación, se expone la Tabla 3 en donde se resumen algunos de los parámetros a considerar, antes de la prescripción del ejercicio deglutorio. Lo anterior, según el músculo y la dispersión de fibras que posea. Es importante destacar que no se describirán cargas y/o tiempos específicos a trabajar (ya que no existe un acuerdo en la literatura para toda la musculatura $\mathrm{CCO}$ ), por lo que solo se harán aproximaciones teóricas, con el fin de acercar al fonoaudiólogo a los parámetros del ejercicio. 
Tabla 3. Parámetro de ejercicio, según músculo y dispersión de fibras.

\begin{tabular}{|c|c|c|c|c|c|c|c|}
\hline Músculo & $\begin{array}{l}\text { Tipo de fibras } \\
\text { preponderantes }\end{array}$ & Estimulo & Carga & Descanso & $\begin{array}{l}\text { Tiempo de } \\
\text { logro }\end{array}$ & $\begin{array}{l}\text { Duración del } \\
\text { efecto }\end{array}$ & Fatiga \\
\hline Cricofaríngeo & $\begin{array}{l}\text { Lenta } \\
\text { (I y IIA) }\end{array}$ & ++ & -- & -- & ++ & ++ & -- \\
\hline Temporal & $\begin{array}{l}\text { Intermedia } \\
\text { (IIA y I) }\end{array}$ & + & $+/-$ & - & + & $+/-$ & $+/-$ \\
\hline Constrictor de la faringe & Principalmente & & & & & & \\
\hline Milohioideo & Rápida & - & + & + & - & - & + \\
\hline Geniogloso & (IIA y IIX) & & & & & & \\
\hline $\begin{array}{l}\text { Digástrico } \\
\text { Orbicular de los Labios }\end{array}$ & $\begin{array}{l}\text { Rápida } \\
\text { (IIX y IIA) }\end{array}$ & -- & ++ & ++ & -- & -- & ++ \\
\hline
\end{tabular}

Simbología: ++ = Alto; + = Moderado; +/- = Medio; - = Leve; -- = Bajo o nulo.

\section{RELEVANCIA CLÍNICA PARA EL QUEHACER FONOAUDIOLÓGICO}

La tendencia mundial señala que el ejercicio fonoaudiológico deberá estar basado en el cotejo de variables objetivas y en la desestimación de las mediciones perceptuales que requieren de la experiencia del evaluador. Ello hace necesario que el profesional fonoaudiólogo conozca las distintas características de las fibras musculares, previo a la elaboración de ejercicios en la zona CCO.

El conocimiento sobre el efecto del ejercicio muscular de la zona CCO se encuentra aún en crecimiento. Por ejemplo, recientemente se demostró que no solo la edad y el sexo son factores para considerar en la prescripción de ejercicios deglutorios, sino también las experiencias musculares en tronco y extremidades se relacionan con el ejercicio muscular CCO (VanRavenhorst-Bell et al., 2018). VanRavenhorst-Bell et al. (2018) reportaron que la fuerza muscular de la lengua varía según el tipo de deporte que realice una persona. Así, aquellos que efectúan levantamiento de pesas tienen una mayor presión lingual que las personas corredoras ( $63 \mathrm{kPa}$ en los levantadores de pesas, versus $55 \mathrm{kPa}$ en los corredores). La duración de la contracción (resistencia) también muestra resultados distintos, ya que los corredores tienden a mantener por mayor tiempo la contracción (23 segundos los corredores, versus 10 segundos los levantadores de pesas). Toda esta información muestra que las personas corredoras tienen más fibras tipo I (permitiéndoles tener alta resistencia, pero baja fuerza), en contraposición con los levantadores de pesas, que poseen más fibras tipo II (permitiéndoles tener más fuerza, pero baja resistencia). Ello demuestra que el profesional fonoaudiólogo necesita estar constantemente actualizando sus conocimientos sobre el trabajo a nivel de la musculatura $\mathrm{CCO}$.

Por otra parte, se sabe que el ejercicio deglutorio por sí solo, cualquiera sea este, es insuficiente para demostrar resultados. Por ello, cada vez se debe dar respuesta a los parámetros del ejercicio (Carnaby et al., 2019). Consecuentemente, el conocimiento acabado de la fisiología del ejercicio oral será de gran relevancia en la disminución de tiempos de tratamiento y la fundamentación de la intervención en la deglución, con protocolos más eficaces. Lo anterior debido a que se considerará no solo la anatomía del músculo, sino las fibras que la componen, y cómo debería ser el tipo de ejercicio, según la dispersión de estas.

\section{REFERENCIAS}

Adams, V., Mathisen, B., Baines, S., Lazarus, C., \& Callister, R. (2013). A systematic review and meta-analysis of measurements of tongue and hand strength and endurance using the Iowa Oral Performance Instrument (IOPI). Dysphagia, 28(3), 350-369. https://doi.org/10.1007/s00455-013-9451-3

Bottinelli, R., \& Reggiani, C. (2000). Human skeletal muscle fibres: Molecular and functional diversity. Progress in Biophysics and Molecular Biology, 73(2-4), 195-262. https://doi.org/10.1016/s0079-6107(00)00006-7

Canepari, M., Pellegrino, M. A., D’Antona, G., \& Bottinelli, R. (2010). Skeletal muscle fibre diversity and the underlying mechanisms. Acta Physiologica (Oxford, England), 199(4), 465-476. https://doi.org/10.1111/j.1748-1716.2010.02118.x

Carnaby, G. D., LaGorio, L., Silliman, S., \& Crary, M. (2019). Exercise-based swallowing intervention (McNeill Dysphagia Therapy) with adjunctive NMES to treat dysphagia post-stroke: A double-blind placebo-controlled trial. Journal of Oral Rehabilitation, 47(4), 501-510. https://doi.org/10.1111/joor.12928 
Chu, S. Y., \& Barlow, S. (2016). A Call for Biomechanics to Understand Hypotonia and Speech Movement Disorders in Down Syndrome. En Advances in Communication Disorders (pp. 2-40). Avid Science Publications.

Cichero, J., \& Murdoch, B. (Eds.). (2006). Dysphagia: Foundation, Theory and Practice. John Wiley \& Sons.

Crary, M. A., Carnaby, G. D., LaGorio, L. A., \& Carvajal, P. J. (2012). Functional and physiological outcomes from an exercise-based dysphagia therapy: A pilot investigation of the McNeill Dysphagia Therapy Program. Archives of Physical Medicine and Rehabilitation, 93(7), 1173-1178. https://doi.org/10.1016/j.apmr.2011.11.008

Dietsch, A. M., Clark, H. M., Steiner, J. N., \& Solomon, N. P. (2015). Effects of Age, Sex, and Body Position on Orofacial Muscle Tone in Healthy Adults. Journal of Speech, Language, and Hearing Research: JSLHR, 58(4), 1145-1150. https://doi.org/10.1044/2015_JSLHR-S-14-0325

Eriksson, P. O., \& Thornell, L. E. (1983). Histochemical and morphological muscle-fibre characteristics of the human masseter, the medial pterygoid and the temporal muscles. Archives of Oral Biology, 28(9), 781-795. https://doi.org/10.1016/0003-9969(83)90034-1

Fuller, D., Pimentel, J., \& Peregoy, B. (2012). Applied Anatomy and Physiology for Speech-Language Pathology and Audiology.

Hwang, K., Huan, F., \& Kim, D. J. (2011). Muscle fiber types of human orbicularis oculi muscle. The Journal of Craniofacial Surgery, 22(5), 1827-1830. https://doi.org/10.1097/SCS.0b013e31822e8468

Kent, R. (2010). Muscle-fiber heterogeneity in craniofacial muscles: Implications for speech development and speech motor control [Poster]. Motor Speech Conference, Savannah, Georgia: University of Wisconsin - Madison. https://academic.oup.com/ejo/article-lookup/doi/10.1093/ejo/cjp093

Korfage, J. A., Brugman, P., \& Van Eijden, T. M. (2000). Intermuscular and intramuscular differences in myosin heavy chain composition of the human masticatory muscles. Journal of the Neurological Sciences, 178(2), 95-106. https://doi.org/10.1016/s0022-510x(00)00372-5

Kristmundsdottir, F., Mahon, M., Froes, M. M., \& Cumming, W. J. (1990). Histomorphometric and histopathological study of the human cricopharyngeus muscle: In health and in motor neuron disease. Neuropathology and Applied Neurobiology, 16(6), 461-475. https://doi.org/10.1111/j.13652990.1990.tb01286.x

Leese, G., \& Hopwood, D. (1986). Muscle fibre typing in the human pharyngeal constrictors and oesophagus: The effect of ageing. Acta Anatomica, 127(1), 7780. https://doi.org/10.1159/000146241
López, J., \& Fernández, A. (2006). Fisiología del Ejercicio. Médica Panamericana. https://www.medicapanamericana.com/mx/libro/fisiologia-delejercicio-incluye-version-digital

Martín, M. C. (1999). Caracteristicas Neuromusculares de la mordida cruzada posterior unilateral: Estudio electromiográfico, kinesiográfico y tomografico [Tesis Doctoral, Universidad Complutense de Madrid]. https://eprints.ucm.es/3129/1/T23301.pdf

McArdle, W. D., Katch, F. I., \& Katch, V. L. (2010). Exercise Physiology: Nutrition, Energy, and Human Performance. Lippincott Williams \& Wilkins.

Osterlund, C., Thornell, L.-E., \& Eriksson, P.-O. (2011). Differences in fibre type composition between human masseter and biceps muscles in young and adults reveal unique masseter fibre type growth pattern. Anatomical Record (Hoboken, N.J.: 2007), 294(7), 1158-1169. https://doi.org/10.1002/ar.21272

Saigusa, H., Niimi, S., Yamashita, K., Gotoh, T., \& Kumada, M. (2001). Morphological and histochemical studies of the genioglossus muscle. The Annals of Otology, Rhinology, and Laryngology, 110(8), 779-784. https://doi.org/10.1177/000348940111000815

Sciote, J., Horton, M., Rowlerson, A., \& Link, J. (2003). Specialized Cranial Muscles: How Different Are They from Limb and Abdominal Muscles? Cells, tissues, organs, 174(1-2), 73-86. https://doi.org/10.1159/000070576

Stone, M., \& Murano, E. (2007). Speech patterns in a muscular hydrostat: Lip, tongue and glossectomy movement. Third B-J-K Symposium on Biomechanics, Healthcare and Information Science, 137-154. https://www.researchgate.net/publication/242766760_SPEECH_PATTERNS_IN _A_MUSCULAR_HYDROSTAT_LIP_TONGUE_AND_GLOSSECTOMY_M OVEMENT

Sundman, E., Ansved, T., Margolin, G., Kuylenstierna, R., \& Eriksson, L. I. (2004). Fiber-type composition and fiber size of the human cricopharyngeal muscle and the pharyngeal constrictor muscle. Acta Anaesthesiologica Scandinavica, 48(4), 423-429. https://doi.org/10.1111/j.1399-6576.2004.00364.x

Susanibar, F., Dioses, A., \& Monzón, K. (2016). El habla y otros actos motores orofaciales no verbales: Revisión Parte II. Revista Digital EOS, 68-105. https://franklinsusanibar.com/el-habla-y-otros-actos-motores-orofaciales-noverbales-revision-parte-ii/

VanRavenhorst-Bell, H., Coufal, K., Patterson, J., \& Mefferd, A. (2018). A comparative study: Tongue muscle performance in weightlifters and runners. Physiological Reports, 6(22), e13923. https://doi.org/10.14814/phy2.13923 